\title{
A INFORMAÇÃO CIENTÍFICA E TECNOLÓGICA BRASILEIRA NO ÂMBITO DA SOCIEDADE DA INFORMAÇÃO: UMA ANÁLISE DAS INICIATIVAS GOVERNAMENTAIS
}

\author{
Fábio Mascarenhas e Silva
}

\section{Resumo:}

Aborda as iniciativas governamentais referentes à gestão da Informação Científica e Tecnológica (ICT) brasileira no âmbito da Sociedade da Informação a partir da leitura crítica de quatro publicações oficiais (Livro Verde da Sociedade da Informação no Brasil; Livro Branco: Ciência, Tecnologia e Inovação; Ciência e Tecnologia para a construção da Sociedade da informação; e Contribuição para políticas de Informação Científico-Tecnológica). Traz ainda um breve retrospecto histórico da comunicação científica e, mais restritamente, da política brasileira da ICT.

\section{Palavras-chave:}

Sociedade da informação; Informação científica e tecnológica; Políticas públicas; Livro verde; Livro branco

\section{THE BRAZILIAN SCIENTIFIC AND TECHNOLOGICAL INFORMATION IN THE SCOPE OF THE IFORMATION SOCIETY: THE GOVERNMENTAL INITIATIVES' ANALYSIS}

\begin{abstract}
:
This article discuss the Brazilian governmental initiatives in Scientific and Technological Information management in the Information Society to arise from critical reading of four official publications (Green Book of the Information Society of Brazil; White Book: Science, Technology and Innovation; Science and Technology for the construction of the Information Society; and Contribution for policies of Scientific Information). It

also brings a brief historical approach of scientific communication and Brazilian Scientific and Technological Information management.
\end{abstract}

Key Words:

Information society; Scientific and technological information management; Public policy; Green book; White book 


\section{INTRODUÇÃO}

Diz-se que estamos vivendo numa 'Sociedade da Informação', mas há quem prefira chamá-la de Sociedade do Conhecimento ou Sociedade pós-industrial. Porém, discutir nomes diferentes para um mesmo significante não importa para o escopo das nossas reflexões, uma vez que não contemplaremos as variações de um mesmo tema, porém o posicionamento da política nacional no que respeita à gestão da Informação Científica e Tecnológica (ICT) nesse (dito) novo modelo de sociedade. E, visando evitar confusões terminológicas, optamos por adotar o termo Sociedade da Informação (SI) por ser este o mesmo utilizado nos documentos oficiais analisados.

Destacamos inicialmente a criação do "Programa Sociedade da Informação no Brasill" em 1996 que resultou na publicação do Livro Verde (2000). Para Sardenberg (TAKAHASHI, 2000, p. 5) o Livro Verde constituiu um conjunto de propostas visando impulsionar “a Sociedade da Informação no Brasil em todos os seus aspectos: ampliação do acesso, meios de conectividade, formação de recursos humanos, iniciativa à pesquisa e desenvolvimento de nossas aplicações". Se concretizadas, estimava-se que essas ações resultassem em benefícios sociais como a introdução das tecnologias de informação no ambiente empresarial acompanhada da inclusão das classes menos favorecidas.

Contudo, nossos indicadores sociais revelam uma realidade incompatível com o anseio de sermos uma sociedade favorecida pelos avanços do conhecimento. Problemas recorrentes como os de educação, saúde e moradia, não parecem estar atrelados aos avanços científicos, tecnológicos e inovativos nacionais. Porém, analisar um contexto tão amplo expandiria o debate para questões que extrapolariam a nossa pretensão em discutir essencialmente as proposições relacionadas à gestão da ICT do Brasil numa SI. Assim, em nossas colocações, enfatizaremos o conteúdo de quatro publicações governamentais: Ciência e Tecnologia para a construção da Sociedade da informação; Sociedade da Informação no Brasil: Livro Verde; Livro Branco: Ciência, Tecnologia e Inovação; e Contribuição para políticas de Informação Científico-Tecnológica.

Ressaltamos que o nosso interesse em debater estas questões surgiu a partir da leitura da primeira diretriz estratégica do Livro Branco (2002, p. 50-53): "Implantar um efetivo Sistema Nacional de Ciência, Tecnologia e Inovação”. E, cientes de que a produção, distribuição e difusão do conhecimento - sobretudo no ambiente acadêmico e empresarial - são condições sine qua non para se alcançar tal diretriz, sentimo-nos motivados a explorar o tema sabendo que a ICT brasileira, 
infelizmente, não se demonstra suficientemente habilitada para promover o fluxo dos nossos estoques de informação.

Ainda, também nos intrigou a constante associação entre a SI e as tecnologias da informação, mais precisamente a Internet. Se por um lado reconhece-se que a Internet facilitou o fluxo informacional, por outro, sabe-se que ela, por definição, é basicamente uma rede eletrônica de comunicação. E se estamos falando de ICT, vale lembrar que o diferencial está nos conteúdos transmitidos, sejam através de redes ou de quaisquer outros canais de comunicação. Assim, identificamos a priori uma desatenção às políticas voltadas aos produtores e usuários de estoques de informação especializados.

\section{COMUNICANDO A ICT}

Antes de iniciarmos a análise das publicações, traçaremos sucintamente um histórico do processo de comunicação da informação no contexto científico e tecnológico, que segundo Meadows (1999), teve origens na Grécia Antiga, sendo a Academia o primeiro ambiente destinado à disseminação (oral) das reflexões sobre o mundo. Já a tradição da escrita descende dos discursos de Aristóteles que foram registrados em manuscritos, hábito este que influenciou a cultura árabe e posteriormente a Europa ocidental.

Em seguida, algumas inovações técnicas permitiram que o conhecimento fosse distribuído de forma mais eficiente, não restando dúvidas que a introdução da imprensa na Europa, no século $\mathrm{XV}$, contribuiu consideravelmente para o crescimento das publicações no mundo. Mas foi a partir da criação em 1662 da Royal Society de Londres que a ciência demonstrou uma preocupação efetiva com a comunicação científica, e entre os motivos que contribuíram para a publicação dos primeiros periódicos, destaca-se o interesse dos editores em aferirem lucros e a própria necessidade de uma comunicação mais eficiente possível, para a crescente clientela interessada em novidades.

Depois, as mudanças da própria ciência acarretaram em transformações na sua maneira de compartilhar as contribuições entre seus pares. A esse respeito, Mathias (1972) considera que o avanço do conhecimento científico foi um fenômeno europeu, assim como foi também na Europa principalmente na Inglaterra e França - que se iniciou o vínculo entre a ciência e a técnica, visando popularizar as descobertas no intuito de alcançar resultados práticos na indústria e agricultura. 
Outra questão interessante foi sugerida por Wersig (1993), que analisou algumas mudanças ocorridas no papel do conhecimento, dentre as quais destacamos a fragmentação, que contribuiu para a diversidade e crescimento de produtos informacionais especializados como bases de dados, livros e periódicos.

As publicações técnicas e científicas passaram então a apresentar um crescimento vertiginoso, verificável em Weisman (1972) ao relatar que no início do século XIX existiam cerca de 100 periódicos, em 1830 este número aumentou para 500 e em 1850 registravam-se 1000 títulos. No ano de 1900 o número atingiu 10.000 títulos, e, segundo uma avaliação feita pela Library of Congress dos Estados Unidos, por volta da década de 1960 eram publicados no mundo cerca de 30.000 títulos de periódicos técnicos e científicos.

Atualmente, somente na Science Citation Index (SCI) da base de dados do Institute for Scientific Information (ISI) há 5.000 periódicos indexados (dos quais apenas 15 são brasileiros). E, no Brasil, de acordo com o CNPq (1997), foram publicados de janeiro de 1995 a junho de 1997 mais de 220 mil trabalhos científicos (incluindo artigos, livros, teses, dissertações e trabalhos em eventos) por nossos pesquisadores.

\section{A ICT NO BRASIL}

Aproveitando os dados apresentados sobre a produção científica nacional, passamos a descrever o desenvolvimento histórico das políticas de ICT brasileira, para tanto, usaremos o interessante retrospecto feito por Dias (2001), que abarcou as décadas de 1950 a 1990.

Décadas de 1950/1960 - Início de um processo de institucionalização e intervenção direta do Estado na formulação de uma política de C\&T e de ICT no país. Criou-se o CNPq, o Instituto Nacional de Tecnologia, o IBBD (Instituto Brasileiro de Biblioteconomia e Documentação) e os sistemas nacionais e internacionais de informação especializada. A produção de ICT centrava-se nas universidades, institutos de pesquisa e empresas públicas de pesquisa e desenvolvimento.

Década de 1970 - Implementação de uma política de C\&T baseada nos Planos Nacional de Desenvolvimento (PND). No I PND, além do Banco de Patentes do Instituto Nacional de Propriedade Industrial, foi criado o Sistema Nacional de Informação Científica e Tecnológica 


\section{ARTIGO}

(SNICT), passando o setor de ICT no Brasil a dividir-se em dois vetores: os sistemas surgidos após as iniciativas e esforços de integração e coordenação desenvolvidas pelo IBBD; e os sistemas pertencentes às áreas enfatizadas no planejamento econômico (independente ou paralelo) dos esforços de coordenação de uma política geral de ICT. No II PND, o IBBD passou a se chamar IBICT e teve como missão planejar e coordenar o setor de ICT no país, assumindo várias missões do extinto SNICT. O IBICT inaugurou o primeiro curso de pós-graduação (mestrado) em Ciência da Informação no país.

Década de 1980 - O III PND instituiu o Sistema Nacional de ICT e centros especializados, criou os Sistemas Estaduais de Informação Científica e Tecnológica (SEICT's) e bancos de dados nacionais.

Década de 1990 - Instalação de Núcleos de Informação Tecnológica no país e criação de sistemas e/ou redes responsáveis pelo programa de disseminação da Informação Tecnológica. Desenvolveram-se redes como a ANTARES e a de Documentação do CNI, e houve a propagação de bibliotecas universitárias virtuais com a implementação de programas como o PROBE, SCIELO, SBInet.

\section{ANALISANDO AS PUBLICAÇÕES GOVERNAMENTAIS}

Nota-se que a história da gestão brasileira em ICT foi construída praticamente a partir de políticas e ações públicas, o que justifica a nossa decisão de buscar em publicações governamentais um panorama geral das propostas direcionadas à inserção do Brasil numa SI, que estão em seguida apresentadas segundo a ordem cronológica das respectivas edições.

a) Ciência e Tecnologia para a Construção da Sociedade Informação (1999)

Proposta encaminhada ao Conselho Nacional de Ciência e Tecnologia visando a desenvolver um projeto de amplitude nacional a fim de estabelecer uma infra-estrutura que permitisse a oferta de serviços e aplicações da Sociedade da Informação, tendo "como base o desenvolvimento de uma nova geração de redes Internet" (BRASIL, 1999, p. 5). Depois de estipulado esse "ponto de partida", o documento prossegue defendendo que as tecnologias e infra-estrutura de informação 
"podem contribuir decisivamente para diminuir a atual distância entre estes [países em desenvolvimento] e os países ricos nos campos econômico e sociocultural”.

No entanto, antes desta colocação (questionável), sugere que as estratégias a serem adotadas estão alinhadas com o discurso proferido na International Telecommunications Union em Buenos Aires pelo então vice-presidente americano Al Gore (1994). Este encontro, denominado "Building the Global Information Infrastructure" (GII), sugeriu uma ação conjunta entre países, blocos econômicos e instituições de grande peso político e econômico como o Banco Mundial, no intuito de formular um plano estratégico de dimensão internacional.

No Brasil, o primeiro passo foi governamental, criando uma infra-estrutura nacional de rede para fomentar o desenvolvimento de serviços, aplicações e conteúdos. Em seguida, numa nova fase “orientada para aplicações", contemplou dez objetivos setoriais, dos quais os três primeiros (Ciência, Tecnologia e Educação) seriam os indutores dos outros. Na Ciência e Tecnologia estimou-se "aumentar radicalmente as capacidades de colaboração e condução de experimentos cooperativos por pesquisadores e de disseminação de resultados científicos e tecnológicos, de forma a melhorar o aproveitamento de oportunidades tecnológicas" (BRASIL, 1999, p. 8).

Apesar da disseminação da produção em C\&T ser apontada como um dos pontos mais significativos da proposta, até o momento isso não assegurou que a ICT passasse a ser bem disseminada. E, um dos fatores prejudiciais à comunicação da produção brasileira é a falta de uniformidade dos sistemas de informação, de bibliotecas, centros de documentação e núcleos de informação tecnológica. Pois, as universidades (públicas e privadas) e centros de pesquisa adotaram sistemas que, em muitas das vezes, são incompatíveis entre si, isolando acervos e comprometendo a recuperação da informação em múltiplos estoques. O lançamento do portal CRUESP $^{1}$ é uma exceção. Trata-se de uma iniciativa das universidades públicas do estado de São Paulo (UNICAMP, USP, UNESP) que facilita a busca simultânea em três diferentes grandes bases.

Não apresentaremos outras falhas semelhantes por entender que a discussão poderia tornar-se fatigante. Entretanto, acreditamos que o problema não consiste na carência de recursos tecnológicos da informação, mas de uma política hábil que entenda os diversos sistemas de informação como partes de um conjunto nacional. Sendo que esta é uma das falhas que atinge o nosso Banco Nacional de Teses, que Gaspari (2002), ironicamente tratou como o único banco que

${ }^{1}$ http://www.cruesp.bc.unicamp.br/ 
não consegue obter êxito no Brasil. Posto isso, adiantamos que problemas semelhantes foram identificados também nos documentos que discutiremos a seguir.

b) Sociedade da Informação no Brasil: Livro verde (2000)

Vimos que a iniciativa de construção da infra-estrutura de uma rede mundial de computadores partiu dos Estados Unidos. O Livro Verde esclarece esta questão ao afirmar que o termo "Information Infrastructure" foi criado pelos EUA, ressaltando o aspecto que eles dominavam bem, isto é, a pré-existência de uma plataforma de computação/comunicações. Já o termo "Information Society" deriva da resposta da União Européia ao desafio lançado pelos EUA na National Information Infraestructure (NII) e, em seguida com a GII” (TAKAHASHI, 2000, p. 107). A ênfase em "Information" traduz o viés europeu em favor de aplicações envolvendo aspectos multiculturais e multilingüísticos e mesmo o uso social das tecnologias.

Por não possuir uma infra-estrutura tecnológica apropriada para consolidar a "Infra-estrutura de Informações", o Brasil aproximou-se das políticas adotadas pela Comunidade Européia. Assim, para esses países que não possuíam uma infra-estrutura adequada para receber esta "Sociedade", restou a saída de promover privatizações, sobretudo nas áreas relacionadas à telecomunicação. $\mathrm{O}$ próprio Livro Verde concorda que "a motivação de iniciativas nacionais na linha do que hoje denominamos "Sociedade da Informação" foi explicitamente econômica, ou industrial com um forte viés econômico" (TAKAHASHI, 2000, p. 108).

Todavia, há uma diferença de postura entre os países com a infra-estrutura pronta (EUA, Canadá) e o restante. Nos primeiros, houve um movimento visando consolidar as áreas de informática, redes, telecomunicações, etc, seguindo este sentido: P\&D $\Rightarrow$ INDÚSTRIA $\Rightarrow$ SERVIÇOS. Ao passo que, o segundo grupo, fez um movimento no sentido de "abrir os serviços de telecomunicações" a fim de encurtar o período de acomodação à nova realidade, a seqüência foi então: SERVIÇOS $\Rightarrow$ INDÚSTRIA $\Rightarrow$ P\&D. É por isto que grande parte da infraestrutura tecnológica utilizada na implantação da "Sociedade da Informação" em países como o nosso, originou-se dos núcleos americanos de pesquisa.

Não questionamos a unilateralidade tecnológica, porém o discurso inconsistente de que vivemos numa Sociedade da Informação. Acreditamos que uma sociedade abarca valores, costumes, hábitos, culturas e não apenas uma infra-estrutura tecnológica. Senão, a Sociedade Industrial seria 
chamada da Sociedade do Tear ou das Máquinas. E sabemos que "industrial” era o contexto sóciopolítico, ideológico e econômico que vigorava naquele momento. Para sermos uma sociedade fundamentada na informação, não bastaria o acesso à Internet, mas condições de uso e geração de fluxos informacionais em diversos setores da sociedade, que contribuíssem para o bem estar social de uma maneira geral.

c) Contribuição para políticas de ICT (2001)

Das quatro publicações analisadas, esta, coordenada pelo IBICT, certamente apresenta as ações mais específicas (e coerentes) à gestão da ICT. Antes de apresentá-la, traçaremos algumas considerações, que segundo o referido documento, delimitam o escopo e a abrangência de uma política de ICT, são elas: as mudanças ocorridas na relação entre políticas, modelos de gestão e ICT; o quadro de referência, considerando a ICT no contexto da formação, formulação e implementação de políticas públicas; e, o papel do setor público e privado na produção, disseminação, uso e absorção de ICT nos contextos de inovação.

No último item reconhece-se que, no Brasil, muitas das informações em C\&T - públicas por sua origem, processamento, transmissão ou destinação - encontram-se dispersas ou são de difícil acesso, sendo necessário “criar mecanismos eficazes e eficientes de visibilidade desta produção para garantir a circulação do conhecimento e maximização do seu potencial de absorção e uso pela sociedade" (GOMES, 2001, p.18).

A obra sugere que, pensar em modelos de gestão e formulação de políticas para contextos informacionais multifacetados pressupõe a reflexão e desenvolvimento de metodologias apropriadas para os espaços de conhecimento, a fim de se constituir sistemas capazes de agregar setores sociais envolvidos e dependentes da informação. Neste ínterim, é preciso se pensar em redes e sistemas como instrumentos de mediação, cujas linguagens têm poder de estruturação quando passíveis de compartilhamento. Do ponto de vista político, é preciso superar o desafio da articulação entre os atores envolvidos, respeitando semelhanças e diferenças entre os ambientes.

A publicação ressalta ainda temas como a economia da informação, políticas e marcos legais, comunicação voltada à transferência da informação e diretrizes envolvendo cidadania e inclusão social. Porém, considerando que a referida obra foi concluída há dois anos, não temos motivos 
para acreditar em resultados positivos em curto prazo, pois as ações propostas parecem não terem avançado além do que foi escrito.

d) Livro Branco: Ciência, Tecnologia e Inovação (2002)

O Livro Branco (LB) é o último discutido, e retomando a nossa introdução, lembramos que foi a partir da sua leitura que nos interessamos em abordar o tema proposto. O LB surgiu com o encerramento do ciclo da Conferência Nacional da Ciência, Tecnologia e Inovação, iniciado com a preparação e a publicação do Livro Verde da C\&T e com a própria realização da Conferência em setembro de 2001. Consiste de uma grande agenda que enumera estratégias a serem desenvolvidas até o ano de 2012, sem no entanto, ser exaustiva em nenhuma de suas seções.

Compõe-se de quatro seções assim distribuídas: a primeira examina os desafios para a consolidação de um Sistema Nacional de Ciência, Tecnologia e Inovação; a segunda apresenta os objetivos de uma proposta de política de Ciência, Tecnologia e Inovação para o período de 20022012; a terceira estabelece as bases para a formulação de diretrizes estratégicas a partir do que existe; a quarta explicita um conjunto de diretrizes estratégicas com vistas às ações necessárias para que se alcancem os objetivos sugeridos.

Nessas seções há elementos compatíveis com os interesses da nossa análise, um deles faz menção à limitação brasileira em transformar o conhecimento em inovações que se traduzam posteriormente em conquistas econômicas e sociais. Entendemos que o êxito desta meta está subordinado, entre outras coisas, à capacidade de promover a comunicação entre os atores envolvidos neste processo.

Em outro trecho cita a inovação incremental, que "objetiva a redução de custos de produção e a definição de segmentos de mercado", acreditando que isso pode aprimorar a competitividade dos produtos nos mercados interno e externo. Porém, nem todas as empresas estão em condições de realizá-la, pois, principalmente para as pequenas e médias “os processos de modernização e o acesso à informação tecnológica - elementos básicos para a inovação - são ainda insuficientes, o que ameaça sua sobrevivência em mercados crescentemente competitivos” (BRASIL, 2002, p. 40).

O LB salienta igualmente a necessidade do poder público ofertar uma infra-estrutura que apóie o segmento da Tecnologia Industrial Básica (TIB) englobando a normalização, certificação, 
metrologia, informação tecnológica e propriedade industrial. E diz ser necessário "colocar a informação tecnológica à disposição da empresa e viabilizar sua utilização para facilitar a inovação" (BRASIL, 2002, p. 51). No entanto, complementando este item, coletamos alguns dados de uma pesquisa (CNI;SENAI, 1996) realizada em 1996. Entre alguns itens desfavoráveis, foi verificado que as empresas brasileiras pouco utilizavam as Normas Técnicas (micro 10,5\%; pequena 14,3\%; média 31,7\%; e grande 43,9\%) e os Bancos de Dados de patentes/propriedade industrial (micro 7,7\%; pequena 4,4\%; média 7,7\%; e grande 16,8\%), limitando-se quase sempre aos recursos informacionais disponíveis na própria empresa (micro 59,9\%; pequena 63,0\%; média $65,1 \%$; e grande $72,8 \%$ ).

Infelizmente, percebemos que a infra-estrutura nacional dedicada ao fluxo informacional (produção, comunicação e uso dos estoques) em ICT é precária, desarticulada, e pouco utilizada, tanto do lado da pesquisa quanto no do desenvolvimento de produtos. E reafirmamos que o acesso a redes não assegura o uso dos sistemas, pois ao que parece, a demanda potencial por estoques de ICT - formada em grande parte por pesquisadores de instituições públicas - não é penalizada por uma exclusão tecnológica, e sim pelo desconhecimento dos sistemas de informação disponíveis. Além disto, tais sistemas, por estarem desarticulados e dispersos, passam a exigir dos usuários, maior experiência e habilidade no uso, proporcionando mais obstáculos a serem vencidos.

\section{CONCLUSÃO}

Ainda que se tenha tentado restringir o tema a ser discutido, reconhecemos a superficialidade na análise dos tópicos propostos, pois seguramente se tratam de questões merecedoras de uma exploração mais exaustiva. No entanto, para uma avaliação mais detalhada do assunto seria recomendável o uso de outras literaturas que apresentassem opiniões que contribuíssem ou refutassem as nossas colocações. Entretanto, como as críticas apresentadas enfatizaram documentos oficiais, a importância em limitar-se a esses textos foi seguida desde o início da pesquisa. Com isso esperávamos discutir os documentos a partir dos textos originais evitando paráfrases ou interpretações de terceiros.

E, pautado nessas fontes, foi possível identificar a fragilidade na qual estão sendo desenvolvidas as políticas de gestão em ICT brasileiras. Isso é facilmente verificado por qualquer cidadão interessado em descobrir o quão habilitadas estão as empresas, pesquisadores, e gestores públicos 
brasileiros a ofertarem e/ou buscarem informações de cunho científico, técnico e mesmo inovador no Brasil.

E por mais que essa não represente uma realidade exclusiva dos países periféricos, é provável que as suas conseqüências sejam ainda maiores nas nações caracterizadas pelos escassos recursos destinados a P\&D. Um exemplo típico e corriqueiro em universidades e empresas é a duplicação de esforços, em razão de não se obter - no estágio inicial - informações necessárias e precisas para o planejamento das pesquisas, podendo acarretar na repetição de estudos, produtos e processos já bem avançados em outras instituições. Santos (2001) descreve um caso no qual uma pequena empresa brasileira desenvolveu um garrafão plástico de água mineral mais adequado ao armazenamento e transporte. Entretanto, ao solicitar o privilégio comercial deste produto, descobriu-se que o mesmo havia sido patenteado há mais de 3 anos. Por simples que pareça, o exemplo ilustra um problema facilmente evitado com um sistema de informação em CT mais eficaz.

Por fim, mesmo não desejando encerrar este estudo com uma mensagem pessimista, acreditamos que os dez anos contemplados no Livro Branco não sejam suficientes para efetivarmos as propostas sugeridas, ao menos no que se refere a ICT.

\section{REFERÊNCIAS}

BRASIL. Ministério da Ciência e Tecnologia. Conselho Nacional de Ciência e Tecnologia. Livro Branco: ciência, tecnologia e inovação. Brasília: MCT, 2002.

BRASIL. Ministério da Ciência e Tecnologia. Conselho Nacional de Ciência e Tecnologia. Ciência e Tecnologia para a construção da Sociedade da Informação. Brasília: MCT, 1999.

CONSELHO NACIONAL DE DESENVOLVIMENTO CIENTÍFICO E TECNOLÓGICO (CNPq). A pesquisa no Brasil: Perfil da pesquisa no Brasil e hierarquização dos grupos de pesquisa a partir dos dados do Diretório dos Grupos de Pesquisa no Brasil. Brasília: Ministério da Ciência e Tecnologia, 1997.

CNI; SENAI. Demanda por informação tecnológica pelo setor produtivo: pesquisa 1996. Rio de Janeiro: CNI, DAMPI: SENAI, CIET, 1996.

DIAS, M. M. K. O gerenciamento de unidades de informação tecnológica sob o enfoque da gestão da qualidade: do estudo das percepções e reações dos clientes ao desenho de novas condutas, 2001. Tese (Doutorado) - Escola de Comunicações e Artes da Universidade de São Paulo, São Paulo, 2001. 
GASPARI, E. O único banco que não dá certo é o de teses. Jornal da Ciência, n. 1967, fev. 2002. Disponível em: <http://www.jornaldaciencia.org.br/index.jsp.>. Acesso em: 15 out. 2002.

GOMES, M. N. G. de; CANONGIA, C.(Org.). Contribuição para políticas de ICT. Brasília: IBICT, 2001.

LATOUR, B.; WOOLGAR, S. A vida de laboratório: a produção dos fatos científicos. Rio de Janeiro: Relume Dumará, 1997.

MATHIAS, P. Who unbound Prometheus? In: MATHIAS, Peter (ed.). Science and Society 16001900. Cambridge: Cambridge University Press, 1972, p. 54-79.

MEADOWS, A. J. A comunicação científica. Brasília: Briquet de Lemos, 1999.

MORIN, E. Ciência com consciência. 6. ed. rev. mod. Rio de Janeiro: Bertrand Brasil, 2002.

SANTOS, R. N. M. dos. Informação estratégica e empresa: o discurso à prova dos fatos.

DataGramaZero, jun. 2001, v. 2, n. 3. Disponível em: <

http://www.dgzero.org/jun01/F_I_art.htm>. Acesso em: 10 maio 2003.

TAKAHASHI, T (org.). Sociedade da Informação no Brasil: Livro Verde. Brasília: Ministério da Ciência e Tecnologia, 2000.

WEISMAN, H. M. Information Systems, services and centers. New York: Becker \& Hayes, 1972.

WERSIG, G. Information Science: the study of postmodern knowledge usage. Information Processing and Management, v. 29, n. 2, p. 229-239, 1993. 


\section{Fábio Mascarenhas e Silva}

Professor do curso de graduação em Ciência da Informação com habilitação em Biblioteconomia da PUC Campinas. Doutorando do programa de Pós-graduação em Comunicação/Ciência da Informação da USP E-mail: fabiomascarenhas@yahoo.com.br

Artigo aceito para publicação em: 12/2004 